\title{
Subaortic Membrane in Adult Mimicking Rheumatic Combined Aortic Stenosis and Regurgitation: Role of Transesophageal Echocardiography in Accurate Diagnosis
}

\author{
${ }^{1}$ Indranil Biswas, ${ }^{2}$ Ganesh Kumar M , ${ }^{3}$ Goverdhan D Puri, ${ }^{4}$ Sandeep S Rana
}

\begin{abstract}
Subaortic membrane (SAM), a rare lesion in the adult population is suspected when there is a high-velocity gradient across the aortic valve with normal functioning aortic valve. With rheumatic heart disease forming the leading cause of valvular pathology in the Indian population, SAM can be missed in adult patients with poor transthoracic echocardiography window and can be misinterpreted as rheumatic heart disease. We report a case of SAM causing aortic valve pathology leading to aortic stenosis and regurgitation which was diagnosed accurately using $2 \mathrm{D}$ and $3 \mathrm{D}$ transesophageal echocardiography during the intraoperative period.
\end{abstract}

Keywords: Rheumatic heart disease, Subaortic membrane, Transesophageal echocardiography.

How to cite this article: Biswas I, Kumar MG, Puri GD, Rana SS. Subaortic Membrane in Adult Mimicking Rheumatic Combined Aortic Stenosis and Regurgitation: Role of Transesophageal Echocardiography in Accurate Diagnosis. J Perioper Echocardiogr 2018;6(2):44-47.

Source of support: Nil

Conflict of interest: None

\section{INTRODUCTION}

Subaortic membrane (SAM) or discrete subaortic stenosis (DSS) is a lesion that is more commonly found in the pediatric population and is usually a rare entity in the adult population (incidence: $6.5 \%$ of adult CHD). ${ }^{1}$ It is suspected in adults when continuous wave Doppler of the aortic valve reveals high gradient, but valve morphology does not seem to be stenotic. However, due to increased body mass index or other associated factors,

\footnotetext{
${ }^{1}$ Fellow Resident, ${ }^{2}$ Senior Resident, ${ }^{3}$ Professor and Head, ${ }^{4}$ Professor.

${ }^{1-3}$ Department of Anesthesia and Intensive Care, Postgraduate Institute of Medical Education and Research, Chandigarh, India

${ }^{4}$ Department of Cardiothoracic and Vascular Surgery, Postgraduate Institute of Medical Education and Research, Chandigarh, India

Corresponding Author:Ganesh Kumar M, Senior Resident, Department of Anesthesia and Intensive Care, Postgraduate Institute of Medical Education and Research, Chandigarh, India, e-mail: mganeshkumar19@gmail.com
}

the assessment of the morphology of the aortic valve and the left ventricular outflow tract (LVOT) is often suboptimal and transesophageal echocardiography (TEE) plays an indispensable role in making a true diagnosis. ${ }^{2}$ The membrane, although most commonly are in a form of the fibromuscular ridge that encircles the LVOT, can also involve the base of an aortic valve cusp or the anterior mitral leaflet (AML). The jet effect generated from the membrane causes thickening, retraction and systolic fluttering of the aortic valve cusps that lead to aortic regurgitation. ${ }^{3}$ We describe a case of an adult female who was diagnosed by TTE as having rheumatic aortic valve disease with severe aortic stenosis (AS) and severe aortic regurgitation (AR) and was referred for aortic valve replacement (AVR) surgery. However, on intraoperative TEE, SAM involving the under-surface of aortic valve leading to restricted movement of the right- and noncoronary cusps, causing moderate-to-severe AR as well as severe forward flow gradient at just beneath the aortic valve was found. Inability to delineate the immediate sub valvular pathology by TTE had probably led to the condition being diagnosed as of rheumatic etiology.

\section{CASE DESCRIPTION}

A 52 years old, nondiabetic, nonhypertensive female presented with $\mathrm{h} / \mathrm{o}$ insidious onset, slowly progressive dyspnea for the last 4 years. The dyspnea was NYHA grade II at onset which progressed to Grade III at the time of presenting to our center. She also had 2 episodes of pre-syncopal symptoms at this time. On examination, she had predominantly grade $4 / 6$ ejection systolic murmur at the aortic area. ECG showed left ventricular hypertrophy. Chest X-ray showed borderline cardiomegaly. Transthoracic echocardiography was reported as rheumatic heart disease, severe AS (peak gradient and mean gradient of 80 and $47 \mathrm{~mm} \mathrm{Hg}$, respectively), severe AR, moderate tricuspid regurgitation (TR), severe pulmonary hypertension $(70 \mathrm{~mm} \mathrm{Hg})$, severe left ventricular systolic dysfunction (25\%). Coronary angiography revealed normal coronaries. She was referred for elective aortic valve replacement (AVR) surgery.

On the day of surgery, before induction of general anesthesia, transthoracic echocardiography was 


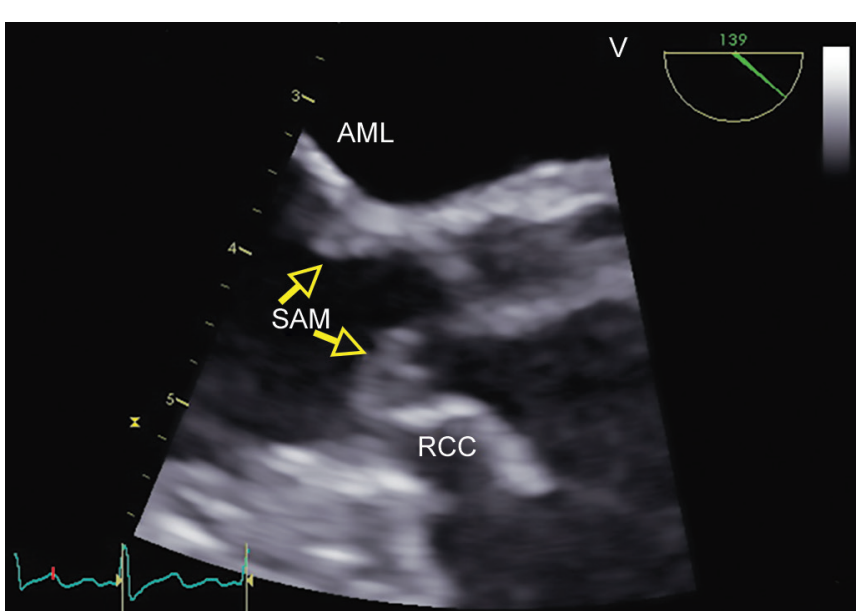

Fig. 1: Mid-esophageal aortic valve long axis view (Zoomed) showing thickened ridge of tissue at the under-surface of the right coronary cusp and also at the base of the anterior mitral valve leaflet. RCC, Right coronary cusp; SAM, Subaortic membrane; AML, Anterior

performed using M5S phased array probe with vivid E9 workstation. However, the window was very poor, preventing adequate visualization of the LVOT region. The aortic valve morphology was also not visualized, although movement of the valve leaflets appeared to be restricted. Significant systolic flow acceleration near the aortic valve and moderate to severe aortic regurgitation was also appreciated. Continuous wave Doppler across the aortic valve revealed significantly elevated gradient (mean gradient: $67 \mathrm{~mm} \mathrm{Hg}$ ). After induction of General Anesthesia, TEE examination was initiated with a 6VT transducer attached with GE vivid E9 workstation. TEE examination (using both short and long axis views in $2 \mathrm{D}$ and also performing $3 \mathrm{D}$ assessment) revealed a thickened ridge of tissue at the under-surface of the right (RCC) and non-coronary cusps (involvement right $>$ left) with significant flow acceleration at the level of the tissue, just proximal to the aortic valve (Fig. 1). The movement of the right coronary cusp was grossly restricted both during systole as well as diastole (Video 1). There was a restricted movement of noncoronary cusp (NCC) as well, although to a lesser extent than that of RCC. Biplane imaging by $2 \mathrm{D}$ and by $3 \mathrm{D}$ examination, the lesion was delineated as a thick fibromuscular membrane involving predominantly the ventricular surface of RCC, extending to the ventricular surface of NCC (RCC $>$ NCC) and also involving the base of the AML (Figs 2 and 3). There was a presence of systolic flow acceleration at the level of this tissue and also the presence of moderate AR (Video 2). Mild concentric hypertrophy with severe LV systolic dysfunction (LVEF: 30\%) was also found.

During surgery, the surgeon confirmed the presence of the subaortic membrane. A small perforation of the Left coronary cusp (LCC) was also found. The subaortic membrane was excised. However, due to extensive
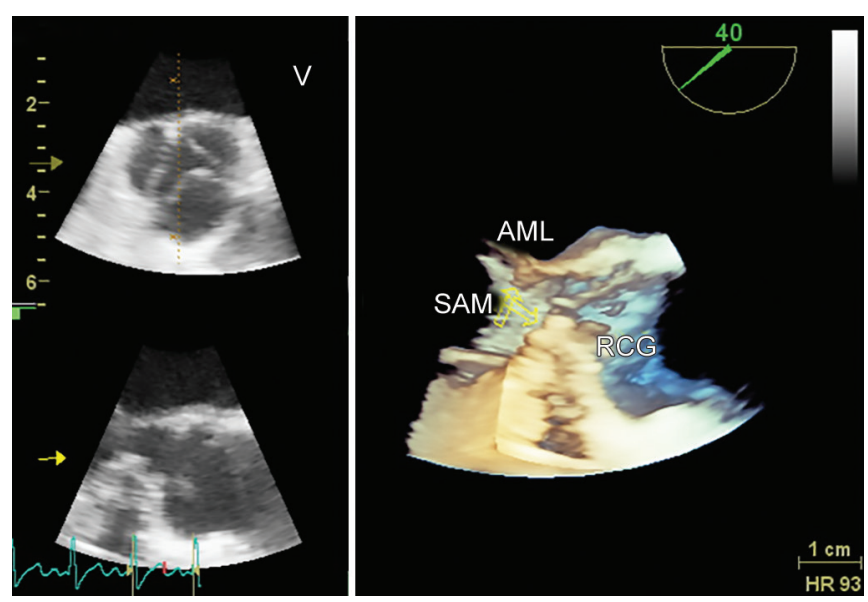

Fig. 2: Mid-esophageal long axis view (3D - Aortic valve zoomed) showing membrane attached to the under-surface of the right coronary cusp and also at the base of the anterior mitral leaflet. RCC, Right coronary cusp; SAM, Subaortic membrane; AML, Anterior

damage and distortion of the aortic valve leaflets, those were excised, and a bi-leaflet mechanical heart valve prosthesis was placed in the aortic position. The patient came off CPB uneventfully and was subsequently weaned off from the ventilator in ICU and was discharged later from hospital uneventfully.

\section{DISCUSSION}

Discrete subaortic stenosis, in a majority, is a lesion that develops in childhood and is usually characterized by unpredictable and often rapid hemodynamic progression and it is commonly associated with aortic regurgitation (30-80\% of patients). ${ }^{4,5}$ Although often considered to be relatively rare in the adult population, the prevalence has been found as $6.5 \%$ of all adult congenital heart diseases. ${ }^{1}$ In adults, the LVOT obstruction usually progresses slowly along several decades and usually, patients become symptomatic by $>50$ years of age1, as was in this case. Adults with DSS almost always have AR $>80 \%$ of

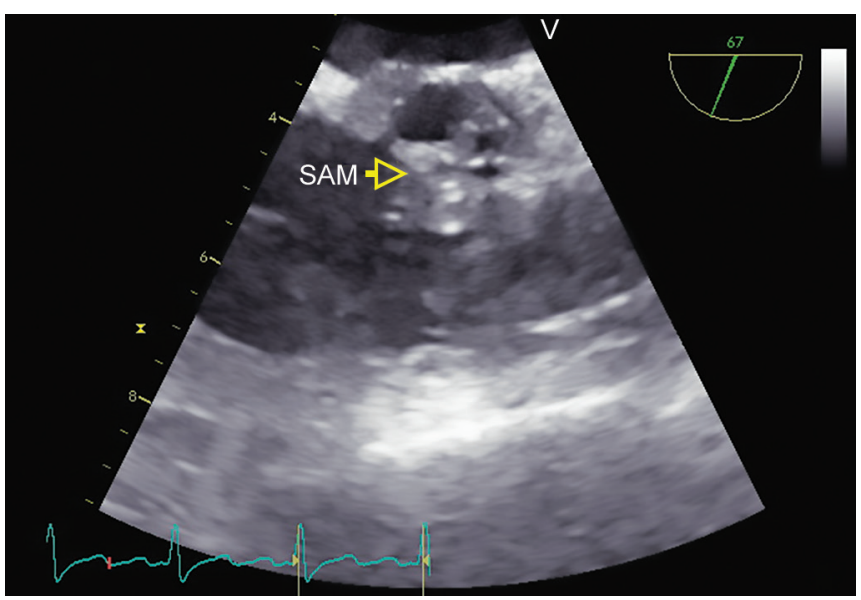

Fig. 3: Mid-esophageal modified aortic valve short axis view at the level inferior to the aortic valve showing membrane predominantly at the region of right coronary cusp 
cases). ${ }^{6}$ The reason behind AR is due to the sub-valvular jet injuring the aortic valve. It can also be due to the involvement of aortic valve cusp by the fibrous tissue. In the present case, the ventricular surfaces of the RCC and NCC were involved, leading to restricted movement of the cusps during both systoles as well as diastole.

Transthoracic echocardiography may be inconclusive about the pathology beneath the aortic valve due to the poor echocardiographic window in patients with higher body mass index (BMI). ${ }^{2}$ The differential diagnosis with hypertrophic obstructive cardiomyopathy (HOCM) can also be difficult because DSS can also have asymmetrical septal hypertrophy and dynamic obstruction of the LVOT. $^{6,7}$

Transesophageal echocardiography plays a valuable role in diagnosing subaortic membrane. ${ }^{8}$ Failure to diagnose subaortic membrane by transthoracic echocardiography followed by diagnosis by TEE has been reported..$^{3,9}$ In most of the previously reported cases, increased gradient upon CW interrogation of the aortic valve along with a normal appearing aortic valve on $2 \mathrm{D}$ examination served as a clue to suspect the presence of a subaortic membrane, which was subsequently confirmed by TEE. In our case, the membrane, due to its location at the undersurface of the RCC and NCC, caused restricted motion of the cusps in both systole and diastole as well. The presence of moderate to severe AR, the presence of significant gradient on $\mathrm{CW}$ interrogation of the aortic valve led to the diagnosis of rheumatic aortic valve disease preoperatively.

In our institution, after the arrival of the patient in the cardiac surgical operating room (OR), TTE is performed routinely before induction of anesthesia and TEE is performed routinely in all cardiac surgical patients after anesthesia induction. In pre-induction TTE, findings were similar to the formal preoperative TTE report. However, the post-induction TEE played a key role in diagnosing the exact pathology, i.e, the subaortic membrane. The clear visualization of the LVOT in multiple planes together with 3D examination established the diagnosis and also delineated the total extent of the membrane. The absence of independent mobility of the tissue and the clinical presentation of the patient indicated that it was unlikely to be vegetation. The TEE findings were reiterated in the surgical findings also. The systolic fluttering of the aortic valve cusps was also visible in our case, as has been mentioned previously by Foker ${ }^{3}$ in his editorial.

In contrary to most of the previous reports where excision of the subaortic membrane was only performed as the aortic valves were structurally normal, the aortic valve in the present case was structurally damaged and distorted with perforation of the LCC as well, which led the surgeon excise all the membranes as well as the aortic valve cusps and replace the valve with a \#17 bi-leaflet mechanical heart valve prosthesis.

DSS is a condition notorious for recurrence. The reported recurrence rates range from 15 to $26 \%$ in various studies. ${ }^{4,10}$ The risk factors for recurrence are (i) preoperative peak gradient $>60 \mathrm{~mm} \mathrm{Hg}$, (ii) distance of SAM from aortic valve $<7 \mathrm{~mm}$, (iii) peeling of the membrane from the aortic valve, (iv) female sex, (v) younger age at operation. ${ }^{3,4}$ Three of the five risk factors were present in the present patient.

Aortic valve replacement in adult patients with SAM may be necessary for patients who present de novo SAM with significant aortic valve dysfunction (significant AR with high LVOT velocities) or for those who had previously undergone SAM resection, but, the disease has recurred and progressed to cause significant $\mathrm{AV}$ dysfunction. ${ }^{11,12}$

\section{CONCLUSION}

Subaortic membrane/discrete subaortic stenosis is a distinct pathology in children as well as adults. The course is often characterized by progressive LVOT obstruction, $\mathrm{AR}$ as well as a predilection for recurrence after corrective surgery. While the diagnosis is often apparent in children by TTE itself, the poor echocardiographic window in higher BMI adult patients often prevents the correct anatomic diagnosis by TTE. TEE, on the other hand, has an excellent ability to visualize the LVOT region. Moreover, availability of 3D Matrix array TEE probes nowadays allows the clinician to view the LVOT and aortic valve region from multiple planes simultaneously and obtain a 3D view as well, which help in establishing the diagnosis of SAM/DSS beyond doubt. It also helps to clearly delineate the extent of the membrane and to find out the involvement of the aortic as well as a mitral valve by it, thus helping to decide the extent of resection needed, to prognosticate about the progress and recurrence of the disease, to decide to repair/replace the aortic valve simultaneously.

Thus, TEE plays a crucial role in the diagnosis of and help in the management of subaortic membrane in adult patients.

\section{REFERENCES}

1. Oliver JM, Gonz_alez A, Gallego P, Sanchez-Recalde A, Benito F, Mesa JM. Discrete subaortic stenosis in adults: increased prevalence and slow rate of progression of obstruction and aortic regurgitation. J Am Coll Cardiol 2001;38:835-842. 
2. Russel David J, Prior David, McLellan Alex. Subaortic Stenosis: What Lies Beneath. Cardiovascular Imaging Case Reports (Article in Press). doi: https://doi.org/10.1016/j.case.2018.01.005

3. John E. Foker. Outcomes and Questions About Discrete Subaortic Stenosis. Circulation 2013;127:1447-1450.

4. Denise van der Linde, Jolien W. Roos-Hesselink, Dimitris Rizopoulos, Helena J. Heuvelman, Werner Budts, Arie P.J. van Dijk, Maarten Witsenburg, Sing C. Yap, Angela Oxenius, Candice K. Silversides, Erwin N. Oechslin, Ad J.J.C. Bogers, Johanna J.M. Takkenberg. Surgical Outcome of Discrete Subaortic Stenosis in Adults: A Multicenter Study. Circulation 2013;127:1184-1191.

5. Rohlicek CV, del Pino SF, Hosking M, Miro J, Côté JM, Finley J. Natural history and surgical outcomes for isolated discrete subaortic stenosis in children. Heart 1999;82:708-713.

6. Chung KJ, Manning JA, Gramiak R. Echocardiography in coexisting hypertrophic subaortic stenosis and fixed left ventricular outflow obstruction. Circulation 1974;49:673-677.

7. Ataul Qureshi, Stephen Awuor, Mathew Martinez. Adult Presentation of Subaortic Stenosis: Another Great Hypertrophic Cardiomyopathy Mimic. Heart, Lung and Circulation 2015;24:e7-e10.
8. Essop MR, Skudicky D, Sareli P. Diagnostic value of transesophageal versus transthoracic echocardiography in discrete subaortic stenosis. Am J Cardiol 1992;70:962-963.

9. Gabriela Dostalova, Zuzana Hlubocka, Tomas Palecek, Vladimír Cerný, Tomas Grus, Jaroslav Lindner, Ales Linhart. Heart Failure Caused by is diagnosed. Subaortic Stenosis in Adulthood: Lesson for Daily Practice. Ann Thorac Surg 2017;104:e91.

10. Hirata Y, Chen JM, Quaegebeur JM, Mosca RS. The role of enucleation with or without septal myectomy for discrete subaortic stenosis. J Thorac Cardiovasc Surg 2009;137:11681172 .

11. Michael Ibrahim, Martin Kostolny, Tain-Yen Hsia, Carin Van Doorn, Fiona Walker, Shay Cullen, Magdi H. Yacoub, Victor T. Tsang. The Surgical History, Management, and Outcomes of Subaortic Stenosis in Adults. Ann Thorac Surg 2012;93:1128 -1133 .

12. John Alfred Carr, Lissa Sugeng, Lynn Weinert, Valluvan Jeevanandam, Roberto M. Lang. Subaortic Membrane in the Adult. Circulation. 2005;112: e347. 